\title{
Chitosan/sulfated locust bean gum nanoparticles: In vitro and in vivo evaluation towards an application in oral immunization
}

\author{
Luis Braz ${ }^{\mathrm{a}, \mathrm{b}, \mathrm{c}, \mathrm{d}, *}$, Ana Grenha ${ }^{\mathrm{d}, \mathrm{e}}$, Domingos Ferreira ${ }^{\mathrm{f}}$, Ana M. Rosa da Costa ${ }^{\mathrm{a}}$, \\ Carlos Gamazo ${ }^{\mathrm{g}}$, Bruno Sarmento ${ }^{\mathrm{h}, \mathrm{i}, \mathrm{j}, \text {,** }}$ \\ a CIQA-Centre of Research in Chemistry of Algarve, Faculty of Sciences and Technology, Campus de Gambelas, 8005-139 Faro, Portugal \\ b School of Health-University of Algarve, Avenida Dr. Adelino da Palma Carlos, 8000-510 Faro, Portugal \\ ' Department of Pharmaceutical Technology, Faculty of Pharmacy, University of Porto, Rua de Jorge Viterbo Ferreira n. ${ }^{\circ} 228,4050-313$ Porto, Portugal \\ d CBMR-Centre for Biomedical Research, University of Algarve, Faculty of Sciences and Technology, Campus de Gambelas, 8005-139 Faro, Portugal \\ e CCMAR-Centre for Marine Sciences, University of Algarve, Campus de Gambelas, 8005-139 Faro, Portugal \\ f UCIBIO, REQUIMTE, Laboratory of Pharmaceutical Technology, Faculty of Pharmacy, University of Porto, Rua de Jorge Viterbo Ferreira, 228, 4050-313 \\ Porto, Portugal \\ ${ }^{g}$ Department of Microbiology, University of Navarra, 31008 Pamplona, Spain \\ h i3S, Instituto de Investigação e Inovação em Saúde, Universidade do Porto, Rua Alfredo Allen, 208, 4200-135 Porto, Portugal \\ i INEB, Instituto de Engenharia Biomédica, Biocarrier Group, Universidade do Porto, Rua Alfredo Allen, 208, 4200-135 Porto, Portugal \\ j CESPU, Instituto de Investigação e Formação Avançada em Ciências e Tecnologias da Saúde, Rua Central de Gandra, 1317, 4585-116 Gandra, Portugal
}

\section{A R T I C L E I N F O}

\section{Article history:}

Received 11 November 2016

Received in revised form

11 December 2016

Accepted 30 December 2016

Available online 31 December 2016

\section{Keywords:}

Locust bean gum

Oral immunization

Polymeric nanoparticles

\begin{abstract}
A B S T R A C T
This work proposes the design of nanoparticles based on locus bean gum (LBG) and chitosan to be used as oral immunoadjuvant for vaccination purposes. LBG-based nanoparticles were prepared by mild polyelectrolyte complexation between chitosan (CS) and a synthesized LBG sulfate derivative (LBGS). Morphological characterization suggested that nanoparticles present a solid and compact structure with spherical-like shape. Sizes around $180-200 \mathrm{~nm}$ and a positive surface charge between $+9 \mathrm{mV}$ and $+14 \mathrm{mV}$ were obtained. CS/LBGS nanoparticles did not affect cell viability of Caco-2 cells after $3 \mathrm{~h}$ and $24 \mathrm{~h}$ of exposure when tested at concentrations up to $1.0 \mathrm{mg} / \mathrm{mL}$. Two model antigens (a particulate acellular extract HE of Salmonella enterica serovar Enteritidis, and ovalbumin as soluble antigen) were associated to CS/LBGS nanoparticles with efficiencies around $26 \%$ for ovalbumin and $32 \%$ for HE, which resulted in loading capacities up to $12 \%$. The process did not affect the antigenicity of the associated antigens. $\mathrm{BALB} / \mathrm{c}$ mice were orally immunized with ovalbumin-loaded nanoparticles $(100 \mu \mathrm{g})$, and results indicate an adjuvant effect of the CS/LBGS nanoparticles, eliciting a balanced Th1/Th2 immune response. Thus, CS/LBGS nanoparticles are promising as antigen mucosal delivery strategy, with particular interest for oral administration.
\end{abstract}

(C) 2017 Elsevier B.V. All rights reserved.

\section{Introduction}

The search for alternative vaccination approaches that may circumvent the limitations of parenteral delivery is not new. Mucosal vaccination has, thus, been gaining popularity in the recent decades and some mucosal vaccines are currently available in the market $[1,2]$. The oral route is the one gathering higher interest concern-

\footnotetext{
* Corresponding author at: School of Health-University of Algarve, Avenida Dr. Adelino da Palma Carlos, 8000-510 Faro, Portugal.

** Corresponding author at: INEB, Instituto de Engenharia Biomédica, Biocarrier Group, Universidade do Porto, Rua Alfredo Allen, 208, 4200-135 Porto, Portugal.

E-mail addresses: lvbraz@ualg.pt (L. Braz), bruno.sarmento@ineb.up.pt (B. Sarmento)
}

ing this alternative vaccination concept, not only because of the general advantages associated with oral administration, but also due to relevant features of the intestine for immunization. In this regard, a special mention is due to the gut associated mucosal tissue (GALT), which has sites where immune responses are initiated and effector sites where adaptive immune responses are executed [2]. The Peyer's patches existing in the GALT comprise the main location of immune cells associated to the intestinal mucosa [3] and are separated from the intestinal lumen by the follicle associated epithelium (FAE) [4]. The FAE is composed of enterocytes, goblet cells and microfold cells (M cells) [3,5], the latter being reported to have a thinner mucus layer, and good ability for antigen uptake and transport to antigen presenting cells (APCs) [6]. Apart from these anatomical specificities, mucosal sites are considered locations of 
primary access for most human pathogens [7], mainly through the mouth. As mucosal immunization is expected to elicit both systemic and mucosal immunity, the development of the latter at the intestinal level becomes a relevant tool towards limiting or preventing pathogen entry, thus inhibiting the consequent infection $[8,9]$.

Notwithstanding the evident ability of the intestinal area for antigen recognition, antigens are biopharmaceuticals, thus being highly sensitive molecules that require specific precautions regarding their formulation and delivery. Indeed, the protein-based structure hinders the possibility of a direct oral administration, mainly because of the low gastric $\mathrm{pH}$ and the high content of proteases $[10,11]$. Suitable particles are therefore demanded for a successful oral immunization approach and nanoparticles have been indicated many times as very useful in mediating this process. Apart from providing the associated antigens with protection from the harsh gastrointestinal conditions referred above, their small size might permit an intimate contact with the epithelial surface, where cells with relevant roles in the generation of an immune response are located [12]. In addition, nanoparticles may also act as immunomodulator adjuvants, meaning that the particle itself mediate the development of the immune response. Thus, the particles may facilitate both the antigen uptake and internalization by GALT and, also, the antigenic cross-presentation by APCs [13]. The use of particle vehicles exhibiting targeting moieties that have a favoured interaction with epithelial glycoconjugates that are specifically activated by pathogens, such as the toll-like receptor (TLR) family or the mannose receptor, has been proposed as a strategy that mimics microbial behaviour in the development of immune responses [13].

The uptake of particles is reported to primarily occur via the $M$ cells [2], which have been referred to provide a privileged contact with mannose residues $[14,15]$ and, therefore, might be used as privileged target for mannose-containing particles. Locust bean gum (LBG) is a polysaccharide of the class of galactomannans, thus having a chemical structure composed of both galactose and mannose units [16] (Fig. 1). Therefore, it potentially has the ability to provide the said favoured contact between nanoparticles and the $M$ cells. The use of mucoadhesive polymers, such as chitosan, may also benefit the contact of nanoparticles with epithelial surfaces, owing to the prolonged retention time provided by mucoadhesion.

On the other hand, inactivated vaccines, including the subunit ones, are gathering higher interest, not only due to the general advantages over the living vaccines, but also because they permit an easier chain of distribution, as a cold-chain is not necessary [17], which is relevant for developing countries [18,19]. Thus, the design of nanoparticles based on LBG and chitosan to be used as antigen delivery systems for oral immunization purposes is presented herein. A negatively charged derivative of LBG was produced (sulfated LBG) to enable the production of nanoparticles by polyelectrolyte complexation. Two different model antigens were associated to the nanoparticles, a liposome-like antigenic complex of Salmonella enterica serovar Enteritidis [20] and ovalbumin, a well-known soluble antigen, in order to carry out immunogenic studies in mice.

\section{Materials and methods}

\subsection{Materials}

Ultrapure chitosan (CS) in the form of hydrochloride salt (Protasan $^{\circledR}$ UP Cl 113, deacetylation degree $=75 \%-90 \%$, molecular weight $<200 \mathrm{kDa}$ ), was purchased from Pronova Biopolymer (Sandvika, Norway). Locust bean gum (LBG) was a kind gift from Industrial Farense (Faro, Portugal). Immunogenic acellular extract obtained from whole Salmonella Enteritidis cells (HE) was prepared according to a previously described method [21]. Ovalbumin (OVA), bovine serum albumin (BSA), phosphotungstate dibasic hydrate, glycerol, phosphate buffered saline (PBS) pH 7.4 tablets, Dulbecco's modified Eagle's medium (DMEM), penicillin/streptomycin (10,000 units/mL, $10000 \mu \mathrm{g} / \mathrm{mL})$, non-essential amino acids, Lglutamine $200 \mathrm{mM}$, trypsin-EDTA solution $(2.5 \mathrm{~g} / \mathrm{L}$ trypsin, $0.5 \mathrm{~g} / \mathrm{L}$ EDTA), trypan blue solution ( $0.4 \%)$, thiazolyl blue tetrazolium bromide (MTT), lactate dehydrogenase (LDH) kit, sodium dodecyl sulfate (SDS), Ponceau $\mathrm{S}$ red staining solution, protease inhibitor cocktail, dimethyl sulfoxide (DMSO), $\mathrm{HCl} 37 \%, \mathrm{H}_{2} \mathrm{O}_{2}$, 4-chloro1-naphtol, $\mathrm{NaCl}, \mathrm{KH}_{2} \mathrm{PO}_{4}$, dialysis tubing (pore size $2000 \mathrm{Da}$ ), $\mathrm{HClSO}_{3}$, dimethylformamide (DMF) and $\mathrm{NaOH}$ were purchased from Sigma-Aldrich (Germany). XT sample buffer, Criterion XT bistris gel, XT MOPS running buffer, Coomassie blue and Tris-glycine buffer were provided by Bio-Rad (USA) and ethanol, PBS-tween (PBS-T) and 3-ethylbenzthiazoline-6-sulfonic acid (ABTS) by VWR (Portugal). Fetal bovine serum (FBS) was obtained from Gibco (USA), molecular mass markers (Novex Sharp Pre-stained Protein Standard) from Invitrogen (Germany). Peroxidase-conjugated goat anti-mouse IgG1, IgG2a and IgA antibodies were purchased from Nordic Immunology (Netherlands) and skimmed milk from Continente (Portugal). Ultrapure water (Mili-Q Plus, Milipore Iberica, Madrid, Spain) was used throughout. All other chemicals were reagent grade.

\subsection{Cell lines}

The Caco- 2 cell line was obtained from the American Type Culture Collection (Rockville, USA) and used between passages 77-93. Cell cultures were grown in $75 \mathrm{~cm}^{2}$ flasks in a humidified $5 \% \mathrm{CO}_{2} / 95 \%$ atmospheric air incubator at $37^{\circ} \mathrm{C}$. Cell culture medium was DMEM supplemented with $10 \%$ (v/v) FBS, 1\% (v/v) L-glutamine solution, $1 \%(\mathrm{v} / \mathrm{v})$ non-essential amino acids solution and $1 \%(\mathrm{v} / \mathrm{v})$ penicillin/streptomycin. Medium was changed every 2-3 days and cells were subcultured weekly.

\subsection{Animals}

The in vivo experiments were performed in strict accordance with good animal practice under the Declaration of Helsinki, the Directive 2010/63/EU and the Portuguese Law DL 113/2013, and in compliance with the regulations of the responsible committee of the University of Algarve (Faro, Portugal). Female BALB/c mice $(20 \pm 1 \mathrm{~g})$ were provided by Instituto Gulbekian de Ciência (Portugal). The animals were starved $7 \mathrm{~h}$ before immunization and only allowed free access to water. After immunization, they were maintained with free access to food and water.

\subsection{Synthesis of sulfated locust bean gum (LBGS) derivative}

A previous purification step of $L B G$ envisaged the removal of the protein content (3-7\%) commonly present in commercial samples [22]. To do so, a standard procedure was followed [23,24], in which LBG $(5.0 \mathrm{~g})$ was slowly dispersed in deionized water $(1000 \mathrm{~mL})$ previously heated to $85^{\circ} \mathrm{C}$, the dispersion being stirred for $1 \mathrm{~h}$. Then, the dispersion was cooled to room temperature and, subsequently, centrifuged $\left(22,000 \times \mathrm{g}, 20^{\circ} \mathrm{C}, 1 \mathrm{~h}\right)$. The supernatant was collected and added to an equal volume of ethanol. The precipitate was collected by vacuum filtration and added again to an equal volume of ethanol. After subsequent collection by vacuum filtration, the precipitate was dried in a vacuum oven at $30^{\circ} \mathrm{C}$ during $72 \mathrm{~h}$ affording $3.9 \mathrm{~g}$ of white powder. The residue was grinded and stored until further use.

Sulfation of LBG was performed by a method established for the sulfation of other polysaccharides [25]. The sulfation agent, 


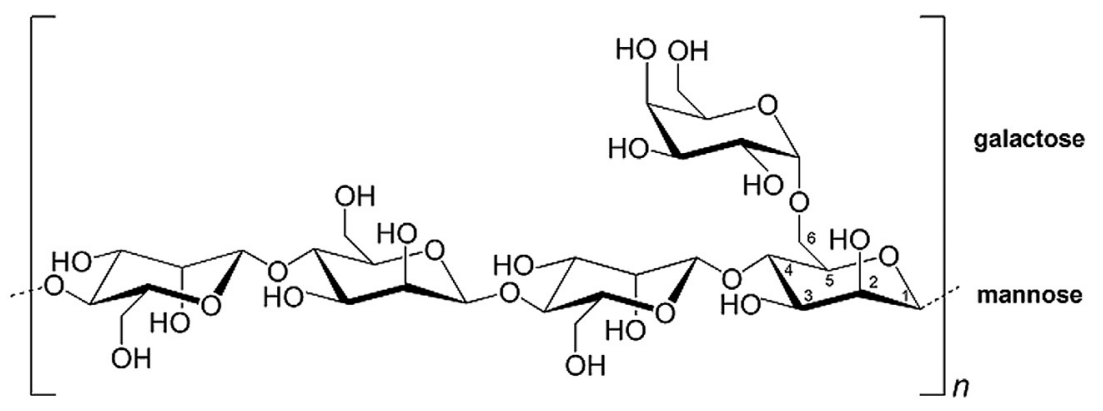

Fig. 1. Chemical structure of locust bean gum.

$\mathrm{SO}_{3} \cdot \mathrm{DMF}$, was prepared by slowly dropping $5 \mathrm{~mL}$ of $\mathrm{HClSO}_{3}$ into $25 \mathrm{~mL}$ of stirred DMF under cooling in an ice water bath, and continuing the stirring for $1.5 \mathrm{~h}$. The obtained solution was stored in the refrigerator until further use.

Purified LBG (500 mg) was slowly dispersed in deionized water $\left(100 \mathrm{~mL}\right.$ ) previously heated to $85^{\circ} \mathrm{C}$, and the dispersion was stirred for $1 \mathrm{~h}$. After that time, the dispersion was cooled to room temperature and poured into an equal volume of ethanol. The precipitate was collected, added to DMF ( $300 \mathrm{~mL})$ and centrifuged $(22000 \mathrm{xg}$, $20^{\circ} \mathrm{C}, 20 \mathrm{~min}$ ). The precipitate was recovered and added to DMF $(100 \mathrm{~mL})$, resting overnight. This dispersion was then filtered and the residue was again added to DMF $(35 \mathrm{~mL})$, this new mixture being stirred at $60^{\circ} \mathrm{C}$ for $30 \mathrm{~min}$, in order to provide the dispersion of LBG into the solvent. Then, the $\mathrm{SO}_{3}$. DMF complex was added $(9.3 \mathrm{~mL})$ and the mixture reacted for $4 \mathrm{~h}$ under magnetic stirring. Subsequently, the mixture was cooled down to room temperature in an ice bath, neutralized with $30 \% \mathrm{NaOH}$ solution until precipitation, and concentrated under reduced pressure at $60^{\circ} \mathrm{C}$ to evaporate the solvent. The residue was dissolved in deionized water $(30 \mathrm{~mL})$ and dialyzed against deionized water $(5 \mathrm{~L})$. The water was changed every $24 \mathrm{~h}$ and, after 3 days, the solution was concentrated under reduced pressure at $40^{\circ} \mathrm{C}$. Then, ethanol was added into the concentrated solution, in order to precipitate the solute, and the dispersion was concentrated under reduced pressure at $40^{\circ} \mathrm{C}$. The previous step was repeated twice, and the last evaporation was performed until full evaporation of the solvent. The obtained powder was dried in a vacuum oven at $40^{\circ} \mathrm{C}$ for 3 days, affording $363 \mathrm{mg}$ of brownish powder that was grinded and stored until further use.

\subsection{Production of chitosan/sulfated locust bean gum (CS/LBGS) nanoparticles}

Different mass ratios of CS/LBGS were tested regarding the preparation of nanoparticles by polyelectrolyte complexation (Table 1). The stock solution of CS, dissolved in ultrapure water, was prepared at a final concentration of $1.0 \mathrm{mg} / \mathrm{mL}$, while LBGS in the same solvent was prepared at $4.0 \mathrm{mg} / \mathrm{mL}$. The solutions were filtered $(0.45 \mu \mathrm{m})$ prior to use. The formulations were prepared by slowly adding $1.8 \mathrm{~mL}$ of LBGS to $1.0 \mathrm{~mL}$ of CS under gentle magnetic stirring at room temperature. Stirring was maintained for $10 \mathrm{~min}$. The concentration of CS was kept constant at $1.0 \mathrm{mg} / \mathrm{mL}$ for the preparation of all formulations, while that of LBGS was modified to obtain the different ratios.

The suspensions of nanoparticles were then centrifuged in eppendorfs with a layer of $10 \mu \mathrm{L}$ of glycerol $(16,000 \times \mathrm{g}, 30 \mathrm{~min}$, $15^{\circ} \mathrm{C}$; Thermo Scientific-Heraeus Fresco 17 , Germany), in order to facilitate the following step of ressuspension. After centrifugation, the supernatants were discarded and nanoparticles were ressuspended with $200 \mu \mathrm{L}$ of ultrapure water.

\subsection{Association of model antigens to CS/LBGS nanoparticles}

\subsubsection{Bacterial antigenic complex}

An antigenic complex, HE, consisting of outer membrane vesicles, was obtained from Salmonella Enteritidis [21]. Its association was performed to the CS/LBGS 1:1.5 and 1:2(w/w) nanoparticle formulations, which selection was mainly driven by the production yield. The stock solution of the HE antigenic complex was prepared by dispersion in ultrapure water $(0.4 \mathrm{mg} / \mathrm{mL})$ using the ultra-sound bath during $15 \mathrm{~min}$ at room temperature. The dispersion was filtered (Millex ${ }^{\circledR}-\mathrm{GV}, 0.22 \mu \mathrm{m}$ low protein binding filter, Millipore, Spain) prior to use. The HE-loaded nanoparticles (NP-HE) were prepared using the same methodology used for the unloaded nanoparticles, but the concentration of LBGS solutions were adjusted using different concentrations of HE dispersions, in order to obtain a theoretical content of HE of $2 \%, 4 \%$ or $8 \%(w / w)$ of the total amount of polymers.

\subsubsection{Ovalbumin}

The association of OVA was performed to the CS/LBGS 1:2(w/w) nanoparticle formulation. The stock solution of OVA was prepared by dissolving it in ultrapure water $(0.3 \mathrm{mg} / \mathrm{mL})$ under magnetic stirring during $15 \mathrm{~min}$ at room temperature. The solution was filtered (Millex ${ }^{\circledR}-\mathrm{GV}, 0.22 \mu \mathrm{m}$ low protein binding filter, Millipore, Spain) prior to use. The OVA-loaded nanoparticles (NP-OVA) were prepared using the same methodology used for the unloaded nanoparticles, but the concentration of LBGS solution was adjusted using the OVA stock solution, in order to obtain a theoretical content of OVA of $8 \%(\mathrm{w} / \mathrm{w})$ of the total amount of polymers.

\subsection{Characterization of CS/LBGS nanoparticles}

\subsubsection{Size, zeta potential and polydispersion index}

The determination of size, zeta potential and polydispersion index (PdI) of nanoparticles was performed on freshly prepared samples. Size and PdI were measured by dynamic light scattering and zeta potential was measured by laser Doppler anemometry, using a Zetasizer Nano ZS (Malvern instruments, UK). To prepare the samples, $20 \mu \mathrm{L}$ of each formulation were diluted in $1 \mathrm{~mL}$ of ultrapure water.

\subsubsection{Production yield}

To determine the production yield of nanoparticles, their preparation was performed as previously described but without the use of the $10 \mu \mathrm{L}$ of glycerol. After discarding the supernatant of each formulation, the pellets were frozen and then dried on a freezedryer (Alpha RVC, Germany). The yield of nanoparticle production (PY) was calculated as follows:

PY $=($ Nanoparticle sediment weight/Total solids weight $) \times 100$ 
Table 1

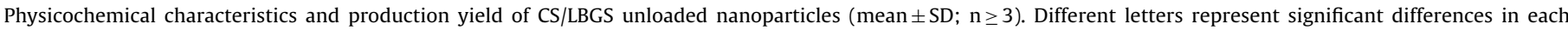
parameter $(\mathrm{P}<0.05)$.

\begin{tabular}{|c|c|c|c|c|}
\hline $\mathrm{CS} / \mathrm{LBGS}(\mathrm{w} / \mathrm{w})$ & Size $(\mathrm{nm})$ & PdI & Zeta potential $(\mathrm{mV})$ & Production yield (\%) \\
\hline $1: 1$ & $174.8 \pm 13.1^{a}$ & $0.10 \pm 0.01$ & $+13.0 \pm 2.7^{b}$ & $9.1 \pm 5.0^{c}$ \\
\hline $1: 1.25$ & $183.0 \pm 4.7^{\mathrm{a}}$ & $0.10 \pm 0.03$ & $+14.5 \pm 1.3^{b}$ & n.d. \\
\hline $1: 1.5$ & $184.0 \pm 4.1^{\mathrm{a}}$ & $0.09 \pm 0.01$ & $+13.0 \pm 0.8^{b}$ & $12.5 \pm 3.9^{c}$ \\
\hline $1: 1.75$ & $182.5 \pm 5.0^{\mathrm{a}}$ & $0.09 \pm 0.01$ & $+13.3 \pm 1.0^{\mathrm{b}}$ & n.d. \\
\hline $1: 2$ & $183.0 \pm 6.1^{\mathrm{a}}$ & $0.13 \pm 0.02$ & $+13.5 \pm 0.6^{b}$ & $30.2 \pm 2.8^{\mathrm{d}}$ \\
\hline $1: 3$ & $198.0 \pm 26.0^{a}$ & $0.12 \pm 0.01$ & $+13.0 \pm 1.0^{b}$ & n.d. \\
\hline $1: 3.25$ & $\mathrm{pp}$ & - & - & - \\
\hline $1: 3.5$ & $\mathrm{pp}$ & - & - & - \\
\hline $1: 4$ & $\mathrm{pp}$ & - & - & - \\
\hline
\end{tabular}

CS: ultrapure chitosan; LBGS: sulfated locust bean gum; n.d.: not determined; PdI: polydispersity index; pp: precipitate.

where nanoparticle sediment weight is the weight after freezedrying and total solids weight is the total amount of solids initially added for nanoparticle formation.

\subsubsection{Morphological analysis}

The morphological examination of CS/LBGS nanoparticles was conducted by transmission electron microscopy (TEM; JEM-1011, JEOL, Japan). The samples were stained with $2 \%(\mathrm{w} / \mathrm{v})$ phosphotungstic acid and placed on copper grids with carbon films (Ted Pella, USA) for TEM observation.

\subsection{Determination of antigen association efficiency}

The amounts of HE and OVA encapsulated in nanoparticles were determined in each sample using the Micro BCA Protein Assay Kit (Pierce, USA). Different calibration curves were performed for each formulation (NP-HE or NP-OVA) using ultrapure water as solvent. Supernatants obtained upon centrifugation of the nanoparticle production media were incubated with the MicroBCA reagent $(2 \mathrm{~h}$, $37^{\circ} \mathrm{C}$ ) in a 96-well plate. After that time, samples were analysed by spectrophotometry (Infinite M200 Tecan, Austria) at $562 \mathrm{~nm}$. The supernatants of unloaded nanoparticles were used for blank correction.

The antigen association efficiency (AE) and loading capacity (LC) were calculated as follows:

$$
\begin{aligned}
& \mathrm{AE}(\%)=[(\text { Total antigen amount }- \text { Free antigen amount }) / \text { Total antigen amount }] \\
& \times 100
\end{aligned}
$$

$\mathrm{LC}(\%)=[$ Total antigen amount - Free antigen amount/Nanoparticle weight $] \times 100$

\subsection{Evaluation of the structural integrity and antigenicity of the loaded antigens}

The integrity of HE and OVA antigens upon association to nanoparticles was confirmed using SDS-PAGE and immunoblotting analysis. Pellets of fresh antigen-loaded nanoparticles (NP-HE and NP-OVA) and free antigens (HE and OVA) were dispersed in electrophoresis sample buffer (XT sample buffer) at a concentration of $1 \mathrm{mg} / \mathrm{mL}$. The mixtures were left in ultrasound bath for $15 \mathrm{~min}$ and then heated at $100^{\circ} \mathrm{C}$ for $10 \mathrm{~min}$. After centrifugation $(16,000 \times \mathrm{g}$, $30 \mathrm{~min}, 15^{\circ} \mathrm{C}$ ) the supernatants were collected and heated at $100^{\circ} \mathrm{C}$ for $10 \mathrm{~min}$. Immunoblotting were performed by using sera from a pool of mice experimentally immunized subcutaneously with either HE $(40 \mu \mathrm{g})$ or OVA $(20 \mu \mathrm{g})$. After SDS-PAGE, the gel was transferred to a nitrocellulose membrane (pore size of $0.45 \mathrm{~mm}$; Whatman $^{\circledR}$, UK) by using a semidry electroblotter (Bio-Rad, USA) at $200 \mathrm{~mA}$ for $30 \mathrm{~min}$, in transfer buffer ( $0.2 \mathrm{M}$ glycine; $24 \mathrm{mM}$ Tris; $20 \%$ methanol, $\mathrm{pH} 8.3$ ). The blot was placed in blocking buffer ( $3 \%$ skimmed milk PBS) overnight at $4{ }^{\circ} \mathrm{C}$. After washing with PBSTween (PBS-T) the blot was incubated with serum diluted 1:100 in PBS-T with $1 \%(\mathrm{w} / \mathrm{v})$ skimmed milk for $3 \mathrm{~h}$. After washing with PBS-T, the blot was incubated for $1 \mathrm{~h}$ at room temperature with the peroxidase-conjugated goat anti-mouse IgG1 (Nordic Immunology) diluted $1: 100$ in PBS-T with $1 \%(w / v)$ BSA. The blot was washed with PBS-T and developed by incubation in a solution containing $\mathrm{H}_{2} \mathrm{O}_{2}$ and 4-chloro-1-naphtol for $3 \mathrm{~min}$ in the dark.

The apparent molecular masses of the proteins present in both antigens were determined by comparing their electrophoretic mobility with that of molecular mass markers.

\subsection{In vitro release in SGF and SIF}

HE and OVA release profiles from CS/LBGS 1:2 (w/w) nanoparticles loaded with $8 \%$ antigen were determined in simulated gastric fluid (SGF) and simulated intestinal fluid (SIF) [26]. In these studies, $2.5 \mathrm{mg}$ of NP-HE and $7.02 \mathrm{mg}$ of NP-OVA were incubated in SGF or SIF $\left(37^{\circ} \mathrm{C}, 100 \mathrm{rpm}\right)$. At appropriate time intervals samples were collected, centrifuged $\left(16,000 \times \mathrm{g}, 10 \mathrm{~min}, 15^{\circ} \mathrm{C}\right)$ and the released HE or OVA determined by the micro BCA (Pierce, USA) assay. Unloaded nanoparticles were submitted to the same conditions and used as blank. All experiments were performed at least in triplicate $(n \geq 3)$.

\subsection{Safety evaluation of unloaded nanoparticles}

The in vitro cell viability and cytotoxicity of CS/LBGS nanoparticles, as well as that of the raw materials involved in nanoparticle production, was assessed by the metabolic assay MTT and the LDH release assay, respectively.

The cells were seeded at a density of $1 \times 10^{4}$ cells/well in 96well microplates, in $100 \mu \mathrm{L}$ of the same medium used for culture in flasks, and were incubated at $37^{\circ} \mathrm{C}$ in $5 \% \mathrm{CO}_{2}$ atmosphere for $24 \mathrm{~h}$ before use. The effect on cell viability induced by three different concentrations $(0.1,0.5$ and $1.0 \mathrm{mg} / \mathrm{mL})$ of unloaded nanoparticles, and raw materials involved in nanoparticle production, was evaluated over $3 \mathrm{~h}$ and $24 \mathrm{~h}$. A SDS solution ( $2 \%, \mathrm{w} / \mathrm{v})$ was used as a positive control of cell death, while cells incubated with DMEM served as negative control. An additional control (DMEM $+\mathrm{H}_{2} \mathrm{O}$ ) consisting in a mixture of DMEM and $\mathrm{H}_{2} \mathrm{O}$ in the same ratio used for the samples was used, in order to evaluate the contribution of materials on cell viability. All formulations and controls were prepared as solution/suspensions in pre-warmed cell culture medium without FBS immediately before application to the cells.

Briefly, culture medium of $24 \mathrm{~h}$-old cells was replaced by fresh medium without FBS containing the test samples or controls. A constant ratio (3:1) between the culture medium and the solution/suspension of the materials was used. After 3 or $24 \mathrm{~h}$ of cell 
exposure, samples/controls were removed and $30 \mu \mathrm{L}$ of the MTT solution $(0.5 \mathrm{mg} / \mathrm{mL}$ in PBS, $\mathrm{pH} 7.4)$ added to each well. After $2 \mathrm{~h}$, any generated formazan crystals were solubilised with $50 \mu \mathrm{L}$ of DMSO. Upon complete solubilisation of the crystals, the absorbance of each well was measured by spectrophotometry (Infinite M200, Tecan, Austria) at $540 \mathrm{~nm}$ and corrected for background absorbance at $650 \mathrm{~nm}$ [27].

The assay was performed at least for three times with six replicates at each concentration of test substance.

Considering the mild effect observed in the MTT assay, the LDH release assay was performed on polymeric solutions and nanoparticle suspensions, after $24 \mathrm{~h}$ exposure to a concentration of $1.0 \mathrm{mg} / \mathrm{mL}$ [28]. Briefly, samples from the culture medium in the seeding plates were centrifuged $\left(16,000 \times g, 5 \mathrm{~min}, 15^{\circ} \mathrm{C}\right)$, and reacted with the LDH release reagent at room temperature and protected from light. The reaction was stopped after $30 \mathrm{~min}$ by adding $15 \mu \mathrm{L} \mathrm{HCl} 1 \mathrm{~N}$. Absorbance was measured by spectrophotometry at $490 \mathrm{~nm}$ with background correction at $690 \mathrm{~nm}$. The relative LDH release (\%) was calculated considering $100 \%$ release for samples incubated with the lysis solution (positive control of cell death) The assay was performed at least three times with three replicates in each experiment.

\subsection{In vivo evaluation of the immune response in $B A L B / c$ mice}

Four groups of six female BALB/c mice were established for the study with NP-OVA. The groups were immunized orally with $200 \mu \mathrm{L}$ of: $100 \mu \mathrm{g}$ of OVA, $100 \mu \mathrm{g}$ of OVA encapsulated in NP (NPOVA); and subcutaneously with $50 \mu \mathrm{L}$ of: $20 \mu \mathrm{g}$ of OVA, $20 \mu \mathrm{g}$ of OVA encapsulated in NP (NP-OVA). Blood and fecal samples were collected at weeks $0,1,2,4$ and 6 post immunization. The fecal pellets were vortexed in PBS with $3 \%(\mathrm{w} / \mathrm{v})$ of skimmed milk $(100 \mathrm{mg} / \mathrm{mL})$ before centrifugation, and then treated with a protease inhibitor cocktail to avoid immunoglobulin degradation.

Specific antibodies (IgG1 and IgG2a from sera; IgA from feces) against OVA were determined by ELISA using 96 microtiter plates (Nunc MaxiSorp, Thermo Scientific). For that purpose, wells were coated overnight with $1 \mu \mathrm{g} / \mathrm{mL}$ of OVA in PBS at $4{ }^{\circ} \mathrm{C}$ and then blocked with $1 \%(\mathrm{w} / \mathrm{v}) \mathrm{BSA}$ (sera samples) or $3 \%(\mathrm{w} / \mathrm{v})$ skimmed milk in PBS-T for $1 \mathrm{~h}$ at room temperature (fecal samples). After washing with PBS-T, a pool of samples was added in twofold serial dilutions in PBS-T starting with $1: 40$, and incubated at $37^{\circ} \mathrm{C}$, for $4 \mathrm{~h}$ (serum); or starting with $1: 1$ and incubated overnight at $4{ }^{\circ} \mathrm{C}$ (feces). Then, washed wells were incubated for $1 \mathrm{~h}$ at room temperature with peroxidase-conjugated goat antibodies anti-mouse IgG1, IgG2a or IgA. For the color development, the substrate-chromogen used was $\mathrm{H}_{2} \mathrm{O}_{2}$-ABTS and after $15 \mathrm{~min}$ (serum) or $30 \mathrm{~min}$ (feces) the absorbance was determined at $\lambda_{\max } 405 \mathrm{~nm}$. The end titers were determined as the dilution of sample giving the mean O.D. $\geq 0.2$ the obtained from untreated mice sera.

\subsection{Statistical analyses}

The $t$-test and the one-way analysis of variance (ANOVA) with the pair wise multiple comparison procedures (Holm-Sidak method) were performed to compare two or multiple groups, respectively. All analyses were run using the SigmaStat statistical program (Version 3.5, SyStat, USA) and differences were considered to be significant at a level of $P<0.05$.

\section{Results and discussion}

\subsection{Characterization of unloaded CS/LBGS nanoparticles}

Several formulations of LBG-based nanoparticles were produced by a very mild polyelectrolyte complexation, a process that involves electrostatic interaction between oppositely charged groups. This procedure takes the advantage of occurring in a hydrophilic environment with mild preparation conditions, avoiding the use of organic solvents or high shear forces that might compromise the stability of encapsulated materials [29,30]. LBG is a natural polymer with neutral charge, which hinders the application of the mentioned methodology to directly produce nanoparticles. In order to overcome that relevant limitation, a sulfate derivative of LBG (LBGS) was produced, exhibiting a negative charge. The derivative was determined to have a C:S molar ratio of 26.76, to which corresponds a degree of substitution of $1.22,0.24$ charges per monomer and a mean molar mass of $932 \mathrm{~g} / \mathrm{mol}$ for the monomer. An $M_{\mathrm{w}}$ of $27 \mathrm{kDa}$ was determined by GPC analysis. Nanoparticles were obtained by complexing LBGS with chitosan (CS). The latter is reported to have a degree of deacetylation of $86 \%$, to which correspond 0.86 positive charges per monomer, and a $M_{\mathrm{w}}$ of $113 \mathrm{kDa}$ [31]. The mean molar mass for the monomer $(198 \mathrm{~g} / \mathrm{mol})$ may be obtained by ponderation of the molar masses of the acetylated and deacetylated units.

Considering the $\mathrm{pH}$ of the involved solutions (4.3 for CS and 5.6 for LBGS), the polymers display positive and negative charges, respectively. When CS and LBGS solutions are mixed, an electrostatic interaction is established between the negatively charged sulfate groups of LBGS and the positively charged amino groups of CS, leading to nanoparticle formation.

As the aim of this work was to disclose the effect of LBGS in the production of nanoparticles, the produced formulations accounted with a similar or higher amount of this polymer comparing with CS. Nine formulations of CS/LBGS nanoparticles were produced with polymeric mass ratios varying within $1: 1$ and $1: 4$. After the preparation procedures, nanoparticles were characterized in terms of size, polydispersion index (PdI), zeta potential and production yield. The detailed results are shown in Table 1.

Formulations with a higher amount of LBGS (ratios $\geq 1: 3.25$, $\mathrm{w} / \mathrm{w}$ ) resulted in precipitation. Considering that a constant amount of CS is used to produce all the formulations, the observed precipitation is possibly due to the presence of an excess of anionic charges, which neutralize CS positive charges and, thus, reduce or eliminate electrostatic repulsion, leading to precipitation. For all the other tested mass ratios, a clear Tyndall effect was observed upon mixing the two polysaccharides, indicating the presence of colloidal particles. Nanoparticles were thus successfully obtained for mass ratios varying between $1: 1$ and $1: 3$. Surprisingly, the size of the particles did not present significant variations among the tested ratios, being in all cases approximately $180 \mathrm{~nm}$. The ratio 1:3 resulted in nanoparticles with an average size of $198 \mathrm{~nm}$, but the standard deviation increased $4-5$ times $(26 \mathrm{~nm})$, which suggests the beginning of the destabilization of the process of nanoparticle formation, which is reinforced by the precipitation occurred in the following ratio $(1: 3.25, \mathrm{w} / \mathrm{w})$. The absence of variations was not expected, as varying the amount of one of the polymers, and therefore the amount of charges, should result in different nanoparticle characteristics. The nanoparticles evidenced a very narrow PdI (around 0.1 ) and a positive zeta potential around $+13 \mathrm{mV}$. As observed for the size, it was also unexpected that the zeta potential did not vary with the alteration of the mass ratios. This effect is better analyzed considering the charge ratios involved in each formulation. By dividing the charge of each repeating unit by its molar mass, a charge per mass ratio is obtained for each polymer. In a $1 / n$ formulation of $C S /$ LBGS, the $-/+$ charge ratio is calculated by:

$-/+$ charge ratio $=n$.charge per mass $(\mathrm{LBGS}) /$ charge per mass $(\mathrm{CS})$

The charge ratio was observed to vary between 0.30 and 0.91 without significant effect on the resulting zeta potential (CS/LBGS nanoparticles $1: 1$ to $1: 3 ; \mathrm{w} / \mathrm{w}$ ). However, on reaching $-/+$ charge ratios of $0.98,1.06$ and 1.21 precipitation occurred (CS/LBGS 
nanoparticles $1: 3.25$ to $1: 4 ; \mathrm{w} / \mathrm{w}$ ). Although this behavior was unexpected, it has been reported in other works regarding polysaccharide nanoparticles produced by the same methodology [32-36].

It was previously demonstrated that the process leading to the assembly of polyelectrolyte complexes has two major steps: the rapid formation of molecular or primary complex particles, and a subsequent phase corresponding to the aggregation of primary particles to secondary particles. While the primary particles are held together by long-range electrostatic interactions, the formation of secondary particles involves short-range dispersive interactions $[37,38]$. In this case, considering the differences between the charge densities of the two polysaccharides ( 0.86 charges/monomer in CS and 0.24 charges/monomer in LBGS), a very inefficient charge pairing should be expected. Therefore, and also regarding the differences in the molecular weights of the polysaccharides, in formulations $1: 1$ to $1: 1.5$, a reduced number of primary complexes should form. The poor charge neutralization should result in enhanced electrostatic repulsion between primary particles, leading to low dispersive attraction and smaller particle sizes, as well as to low yields. By increasing the amount of LBGS, towards the $1: 2$ formulation, a larger number of primary particles form, which should tend to aggregate in larger particles as the $(-1+)$ charge ratio increases to 0.60 . However, that was not the case, with all formulations presenting almost invariant particle sizes, which should mean that more particles formed; thus, the slight increase in yield observed in the latter formulation [38]. The fact that all formulations present similar surface potentials of $\sim 13 \mathrm{mV}$, seems to corroborate this hypothesis, as this potential should correspond to a repulsive electrostatic force overcoming the dispersive interactions and, therefore, preventing the particles from growing further. This behavior was observed in other works [33], normally associated to the use of ultrapure CS. Therefore, another possible explanation may reside in conformational features of CS when the free base form is dissolved in acetic acid or when the hydrochloride salt is dissolved in water.

Taking into account the previous observations regarding the similarity of physicochemical characteristics and the suggested destabilization starting in formulation CS/LBGS 1:3 (w/w), it was decided to determine the production yield of nanoparticles $1: 1$, $1: 1.5$ and $1: 2(\mathrm{w} / \mathrm{w})$. While no significant differences were observed between the first two, with yields of $9-13 \%$, the latter registered a much higher yield around 30\% $(P<0.05)$. This is the trend that is usually observed $[32,39-41]$ and reflects the fact that, up to a certain limit, when increased amounts of LBGS are incorporated, the occurrence of electrostatic interactions is also increased, resulting in the formation of a higher number of nanoparticles [42].

In order to restrict the number of formulations for the subsequent tasks, and considering that the production yield was the most differentiating characteristic of the nanoparticles, it was decided to select the formulations CS/LBGS $1: 1.5$ and $1: 2(\mathrm{w} / \mathrm{w})$ for the rest of the studies. Fig. 2 displays the morphological characterization of representative nanoparticles (CS/LBGS 1:2, w/w). This was performed by TEM and revealed a solid and compact structure, showing a tendency to exhibit a spherical-like shape.

\subsection{Characterization of antigen-loaded nanoparticles}

Bearing in mind the objective of using CS/LBGS nanoparticles in oral vaccination, the first approach regarding the association of an antigen relied on using an immunogenic acellular extract obtained from Salmonella Enteritidis cells (HE), which is a particulate liposome-like antigenic complex [20,43]. After performing the in vivo studies, which results are reported later on, the need to test soluble antigens was identified, in order to permit a clearer evaluation of the real adjuvant properties of the formulated nanoparticles. Therefore, in a second stage of the experiments, the soluble protein

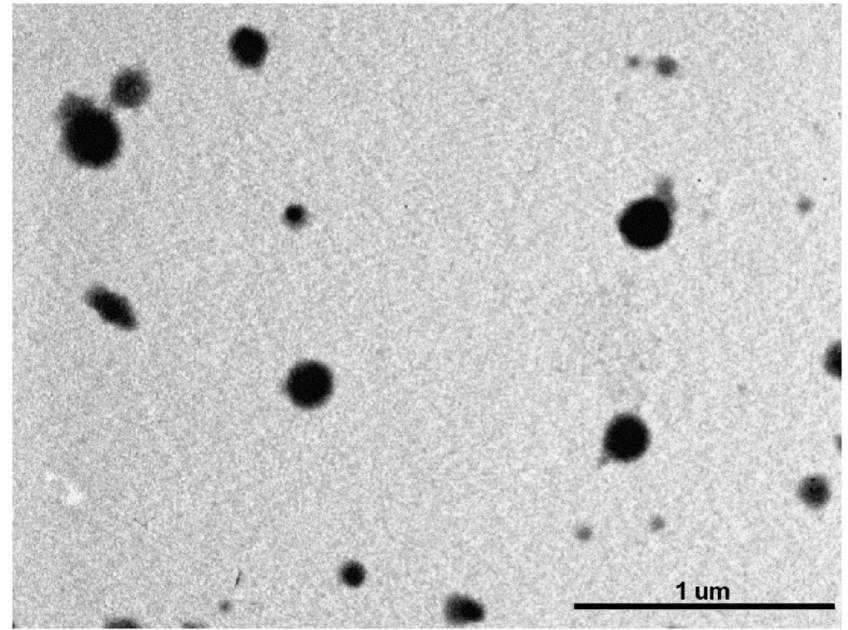

Fig. 2. TEM microphotograph of CS/LBGS 1:2(w/w) nanoparticles.

ovalbumin (OVA) was associated as model antigen. Additionally, as being one of the mostly used molecules for the antigen effect, it enables further comparisons with the literature. As pertinent information, the HE extracts are mainly composed of proteins (29\%) and lipopolysaccharides (LPS, 59\%) [21]. In this context, it is well reported in the literature that LPS fractions have great ability to generate immunological responses, since they are prototypical pathogen-associated molecular patterns (PAMP's) [44]. Therefore, taking into account that OVA is a protein devoid of PAMP components, it is assumed as less immunogenic than the LPS-containing HE.

\subsubsection{Salmonella Enteritidis extract-loaded CS/LBGS nanoparticles}

The first demonstration of the usefulness of the developed nanoparticles to act as adjuvants in a vaccination strategy relies on the determination of their ability to associate antigens. In order to verify this, different amounts of the bacterial HE antigens were associated to nanoparticles CS/LBGS 1:1.5 and 1:2(w/w). Departing from initial concentrations of $2 \%, 4 \%$ and $8 \%(w / w)$ of the total amount of the polymers, an effective and similar association was observed in all cases. In fact, the association efficiency varied within 29-36\% (Table 2), independently of both the initial concentration of antigen and the formulation. These resulted in loading capacities up to $12 \%$. Taking into account that HE is negatively charged when in the LBGS-HE solution, and also considering the high density of free amino groups present in the chitosan solution, it could be assumed that the main factor affecting HE association to the nanoparticles was an electrostatic interaction. This is in agreement with many other works reporting the association of protein-based macromolecules to nanoparticles produced by polyelectrolyte complexation [42,45-47]. In turn, although it could be expected that HE and LBGS might compete in their interaction with chitosan, the obtained results do not show an influence of LBGS content on HE association. This may be due to the fact that LBGS could also interact with HE by means of hydrophobic interactions, hydrogen bonding and other intermolecular forces [39]. The absence of a concentration-dependent effect regarding HE was also unexpected, although it has been observed in other works [41,42].

Table 2 further displays the physicochemical characteristics of HE-loaded CS/LBGS nanoparticles. The size of the particles was around 190-200 $\mathrm{nm}$ independently of the specific formulation and the amount of HE associated. When comparing with the corresponding unloaded nanoparticles, which had a size of $183-184 \mathrm{~nm}$, no significant differences were generally observed. An exception 
Table 2

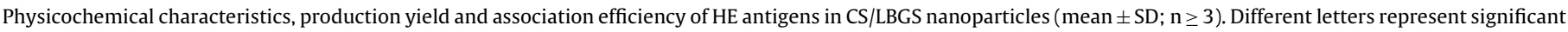
differences in each parameter, evaluated separately for each mass ratio $(P<0.05)$.

\begin{tabular}{|c|c|c|c|c|c|c|c|}
\hline $\mathrm{CS} / \operatorname{LBGS}(\mathrm{w} / \mathrm{w})$ & $\mathrm{HE}(\%)$ & Size $(\mathrm{nm})$ & PdI & Zeta potential (mV) & Production yield (\%) & Association efficiency (\%) & Loading capacity (\%) \\
\hline \multirow[t]{4}{*}{$1: 1.5$} & 0 & $184.0 \pm 4.1^{\mathrm{a}}$ & $0.09 \pm 0.01$ & $+13.0 \pm 0 .^{\mathrm{e}}$ & $12.5 \pm 3.9^{i}$ & - & - \\
\hline & 2 & $191.5 \pm 7.8^{a}$ & $0.11 \pm 0.02$ & $+10.0 \pm 1.4^{\mathrm{f}}$ & $9.6 \pm 6.3^{i}$ & $31.2 \pm 7.4^{\mathrm{k}}$ & $6.4 \pm 1 .{ }^{m}$ \\
\hline & 4 & $190.0 \pm 11.3^{\mathrm{a}}$ & $0.10 \pm 0.01$ & $+10.0 \pm 1.4^{\mathrm{f}}$ & $13.1 \pm 3.8^{i}$ & $29.0 \pm 2.6^{k}$ & $8.5 \pm 0 .{ }^{m}$ \\
\hline & 8 & $196.5 \pm 2.1^{b}$ & $0.08 \pm 0.01$ & $+9.5 \pm 0.7^{\mathrm{f}}$ & $15.5 \pm 0.0^{\mathrm{i}}$ & $25.3 \pm 3.2^{k}$ & $12.1 \pm 1.5^{\mathrm{n}}$ \\
\hline \multirow[t]{4}{*}{$1: 2$} & 0 & $183.0 \pm 6.1^{c}$ & $0.13 \pm 0.02$ & $+13.5 \pm 0 . \mathrm{g}$ & $30.2 \pm 2.8^{j}$ & - & - \\
\hline & 2 & $192.0 \pm 9.9^{c}$ & $0.11 \pm 0.00$ & $+10.5 \pm 0.7^{h}$ & $28.5 \pm 5.2^{j}$ & $35.5 \pm 9.7^{1}$ & $2.4 \pm 0.7^{\circ}$ \\
\hline & 4 & $193.0 \pm 11.3^{c}$ & $0.13 \pm 0.02$ & $+11.5 \pm 0.7^{h}$ & $29.6 \pm 3.9^{j}$ & $34.8 \pm 3.3^{1}$ & $4.5 \pm 0.4^{p}$ \\
\hline & 8 & $202.5 \pm 10.6^{d}$ & $0.13 \pm 0.03$ & $+12.5 \pm 0 . .^{g}$ & $30.1 \pm 3.7^{j}$ & $31.9 \pm 4.0^{1}$ & $7.8 \pm 1.0^{\mathrm{q}}$ \\
\hline
\end{tabular}

CS: ultrapure chitosan; HE: antigenic extract from Salmonella Enteritidis; LBGS: sulfated locust bean gum; PdI: polydispersity index.

was only observed when $8 \%$ HE was associated, for both formulations, with sizes reaching approximately $200 \mathrm{~nm}(P<0.05)$, but this observation is considered to be devoid of physiological relevance. The PdI of the nanoparticles remained remarkably low after association of $\mathrm{HE}$ (around 0.1 ) and a very slight decrease of $2-3 \mathrm{mV}$ in zeta potential was generally observed $(P<0.05)$. Regarding the latter, the only exception was for the formulation $1: 2$, again when $8 \% \mathrm{HE}$ was associated (zeta potential of $+12.5 \mathrm{mV}$ ), in which no significant variation was observed comparing with the equivalent unloaded nanoparticles. Regarding the yield of the process of nanoparticle production, it was also observed an absence of effect upon association of HE antigens, independently of the used concentration. Considering that size and zeta potential values remained approximately similar after the association of the antigen, the maintenance of the production yield is indicative of displacement of the polymers to permit the incorporation of the active molecule.

Taking into account the properties exhibited by HE-loaded nanoparticles, it was decided to select the formulation CS/LBGS 1:2 with $8 \%$ of HE, to perform subsequent studies. This selection was driven by the presence of a higher theoretical amount of LBG and $\mathrm{HE}$ antigens. The morphological examination of this specific formulation was performed by TEM and revealed the maintenance of the solid and compact structure of the unloaded nanoparticles, also showing a tendency to exhibit a spherical-like shape (data not shown).

As shown in Table 2, the developed HE-loaded CS/LBGS nanoparticles evidence adequate physicochemical properties for the objective of oral delivery aimed at mucosal vaccination, with a size around $200 \mathrm{~nm}$ and a positive zeta potential. In fact, the size is small enough to permit an intimate contact with epithelial surfaces, which is maximal at $50-500 \mathrm{~nm}[48,49]$. In turn, the positive zeta potential further potentiates the interaction with epithelia, as this is negatively charged and, thus, an electrostatic interaction is enabled. In summary, these characteristics are expected to provide a prolonged retention of nanoparticles close to epithelial surfaces, potentiating the uptake by $\mathrm{M}$ cells and/or antigen release. The presence of chitosan is expected to further contribute to this effect, owing to its known mucoadhesive ability [50].

\subsubsection{Ovalbumin-loaded CS/LBGS nanoparticles}

As mentioned above, the need to associate a second antigen was identified and OVA was selected for this end. Considering that CS/LBGS nanoparticles 1:2(w/w) containing $8 \%$ HE had been previously selected for further studies, the production of OVAloaded nanoparticles respected the same composition, in order to effectively evaluate the particle contribution as vaccination adjuvant. As stated in Table 3, OVA was successfully encapsulated with a similar efficiency as that for HE (26.4\%), which resulted in a loading of $5.2 \%$. Regarding the physicochemical characteristics of OVA-loaded nanoparticles, there are no statistically significant differences in size when comparing with unloaded particles, while a slight decrease of zeta potential around $4 \mathrm{mV}$ was observed
$(P<0.05)$. The production yield registered a significant increase from $30 \%$ to $38 \%(P<0.05)$. When a comparison with HE-loaded nanoparticles is performed, considering the corresponding formulation, it should be highlighted that OVA-loaded nanoparticles have a significantly lower size (179 nm vs $203 \mathrm{~nm}$ ) and higher production yield $(38 \%$ vs 30\%) $(P<0.05)$. A significant difference was also found for zeta potential, although this only decreased around $3 \mathrm{mV}$ $(P<0.05)$.

Naturally, the literature does not report similar nanoparticles, as LBG is being proposed for the first time herein, but chitosan-based nanoparticles have been suggested many times regarding oral vaccination [51-58]. Occasionally, ovalbumin was the tested antigen [53], resulting in nanoparticle size around $300 \mathrm{~nm}$ and a strong positive zeta potential $(+43 \mathrm{mV})$. As said above, the positive zeta potential is a desirable characteristic to mediate and favor the interaction with the epithelium. In our work, the proposed nanoparticles present a lower zeta potential, but also a lower size, which further benefits this interaction, due to an increased surface area.

\subsection{Evaluation of the structural integrity and antigenicity of the loaded antigens}

As detailed above, the association of either HE or OVA into nanoparticles was performed by means of a mild ionic interaction. Along with the determination of the ability of the developed nanoparticles to associate the selected antigens, it is also of utmost importance to ensure that the particles and the procedure used for their production enable the preservation of the structural integrity and antigenicity of the encapsulated molecules. SDS-PAGE analysis followed by immunoblotting was the method used to perform this evaluation.

The obtained results are depicted in Fig. 3a-d, for HE and OVA, respectively. Regarding HE, SDS-PAGE results shown in Fig. 3a demonstrate that the procedure used for HE entrapment did not affect the structural integrity of the molecules, as no additional fragments are observed in the HE released from nanoparticles (lane 2) when compared with the control HE dispersion (lane 1).

Moreover, the immunoblot shown that the HE-specific antibodies recognized the epitopes in a similar way as for the free HE dispersion (Fig. 3b). This confirms that the antigenicity of HE was not altered after the entrapment into the nanoparticles.

Concerning the association of OVA, similar results were observed. As demonstrated in Fig. 3, the SDS-PAGE of OVA released from nanoparticles (Fig. 3c) showed identical bands for the entrapped (lane 2) and free OVA (lane 1). Furthermore, there were no additional bands indicating the presence of aggregates or fragments greater or less than $45 \mathrm{kDa}$ (molecular weight of OVA). Hence, the data suggest that the structural integrity of OVA was not significantly affected by the entrapment procedure. The antigenicity of OVA was also not modified after association, as the immunoblot bands from OVA solution (Fig. 3d, lane 1) and OVA released from nanoparticles (Fig. 3d, lane 2) were identical. 
Table 3

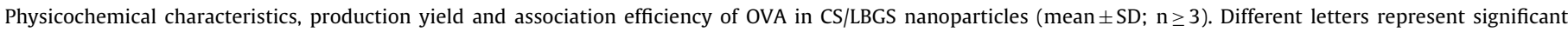
differences in each parameter $(P<0.05)$.

\begin{tabular}{|c|c|c|c|c|c|c|c|}
\hline $\mathrm{CS} / \mathrm{LBGS}(\mathrm{w} / \mathrm{w})$ & OVA (\%) & Size $(\mathrm{nm})$ & PdI & Zeta potential $(\mathrm{mV})$ & Production yield (\%) & Association efficiency (\%) & Loading capacity (\%) \\
\hline \multirow[t]{2}{*}{$1: 2$} & 0 & $183.0 \pm 6.1^{a}$ & $0.13 \pm 0.02$ & $+13.5 \pm 0.6^{b}$ & $30.2 \pm 2.8^{\mathrm{d}}$ & - & - \\
\hline & 8 & $178.6 \pm 6.8^{a}$ & $0.13 \pm 0.02$ & $+9.0 \pm 1.0^{c}$ & $37.9 \pm 4.1^{\mathrm{e}}$ & $26.4 \pm 3.8$ & $5.2 \pm 0.7$ \\
\hline
\end{tabular}

CS: ultrapure chitosan; LBGS: sulfated locust bean gum; OVA: ovalbumin; PdI: polydispersity index.

a)

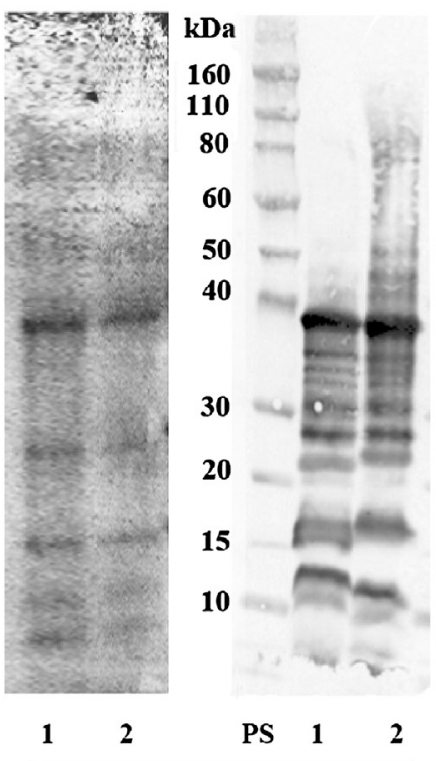

HE c)

d)

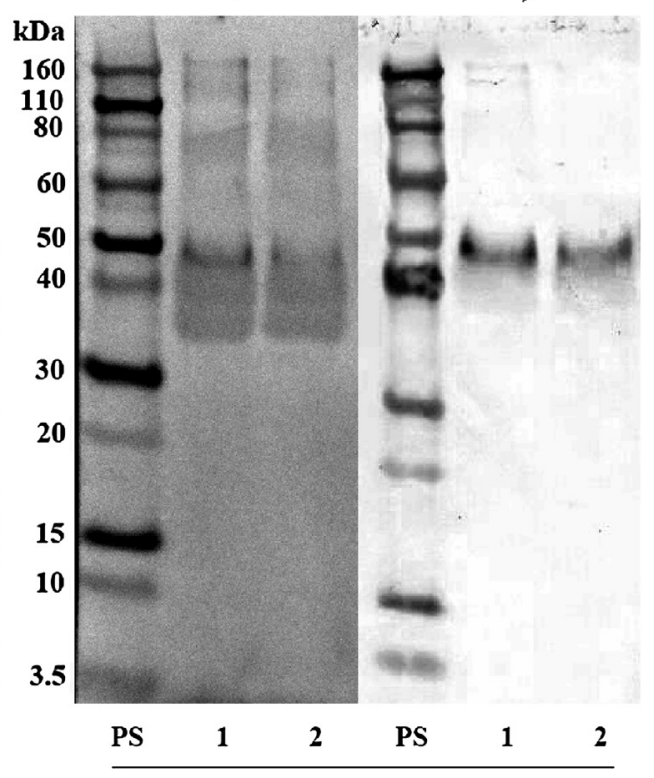

OVA

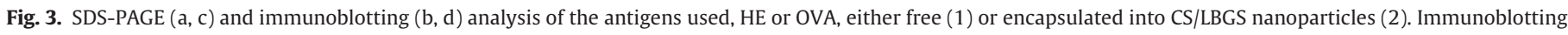
were performed with specific antibodies against HE or OVA, respectively; PS: protein standard.

Altogether, these results indicate the adequacy of polyelectrolyte complexation as a method to produce antigen-loaded polymeric nanoparticles, reinforcing results already available in the literature. In fact, several works reporting the preparation of nanoparticles using methodologies involving electrostatic interaction and using materials such as chitosan, its derivatives and alginate, have demonstrated to provide protection to various model antigens including inactivated influenza virus [59], bovine serum albumin [60], tetanus toxoid [61] and diphtheria toxoid [62].

\subsection{In vitro release in SGF and SIF}

Considering an application in oral vaccination, it is adequate to determine the release of the encapsulated antigens in media simulating both the gastric (SGF) and intestinal (SIF) environments. In the proposed approach it is important that the nanoparticles not only provide protection to the antigens regarding the harsh conditions of the gastric medium, but also prevent their release, in order to maximize the antigen internalization by the $\mathrm{M}$ cells mediated by the particle.

Fig. 4 shows the release profile of HE and OVA in SGF and SIF. As can be observed, HE is considered to not present significant release in any of the tested media. In fact, in SGF it releases a maximum amount of $7.5 \%$ in $2 \mathrm{~h}$, while releasing $4.3 \%$ in SIF after $4 \mathrm{~h}$. Moreover, although it might not be relevant from a physiological point of view, $\mathrm{HE}$ release at the end of $24 \mathrm{~h}$ was $21.6 \% \pm 2.0$ and $13.2 \% \pm 4.8$ in SGF and SIF, respectively. In turn, OVA presented a rather different behavior. In this case, the release in SGF was of $45.0 \%$ at the end of $2 \mathrm{~h}$, and $3.0 \%$ in SIF at the end of $4 \mathrm{~h}$.

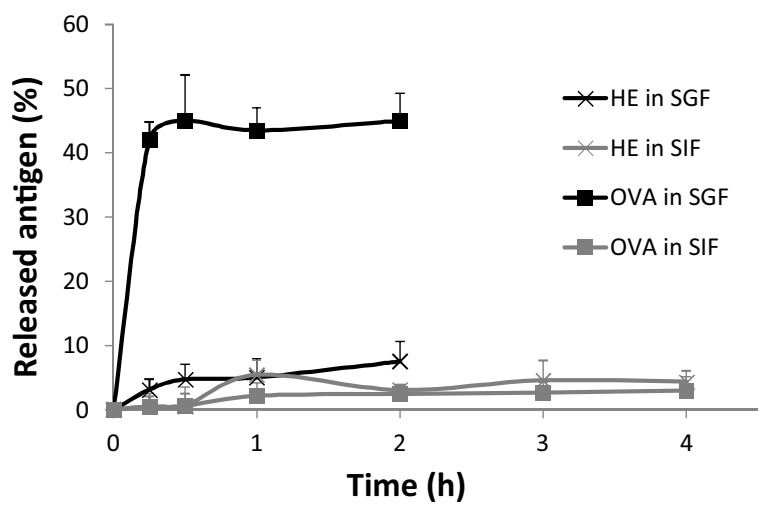

Fig. 4. Antigen released overtime from CS/LBGS nanoparticles in simulated gastric fluid (SGF) and simulated intestinal fluid (SIF), at $37^{\circ} \mathrm{C}($ mean $\pm S D ; n \geq 3)$.

Considering what was stated above, in the case of OVA-loaded nanoparticles, it could be assumed that a high amount of encapsulated OVA releases in the harsh conditions of gastric environment, affecting the biological activity of the molecule. This difference observed for HE-loaded and OVA-loaded nanoparticles could be hypothetically explained by a greater association of HE into the particle, contrasting with a substantial adsorption of OVA onto its surface. Although the determined physicochemical properties do not confirm these effects, it should be reminded that a very small amount of protein is being associated (theoretical load of $8 \%(w / w)$ ). OVA release at the end of $24 \mathrm{~h}$ was $75.5 \% \pm 13.1$ and $9.8 \% \pm 1.5 \mathrm{in}$ SGF and SIF, respectively. It is remarkable that an insignificant amount 


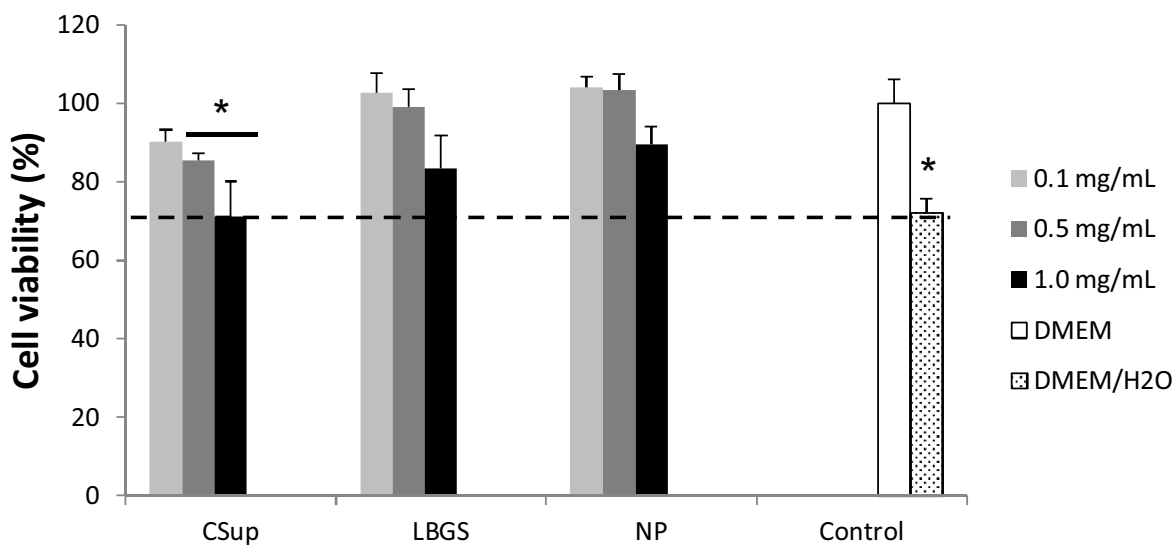

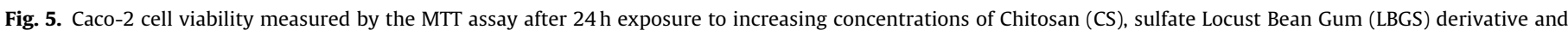

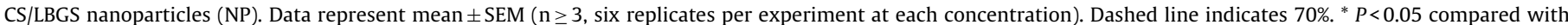
DMEM.

of antigen was observed to be released in SIF in both cases. This means that a great amount of antigen remains associated to the nanoparticles until they reach the contact with the Peyer patches, where the nanoparticles are expected to have a favored contact with the M cells, which will potentiate the immunological response.

Salman et al. studied the HE release from Gantrez ${ }^{\mathbb{B}}$ AN nanoparticles, registering $10 \%$ release in SGF (after $30 \mathrm{~min}$ ) and $12 \%$ in SIF (after $3 \mathrm{~h}$ ) [63], values slightly higher than those obtained in our study. In turn, Garinot et al. studied the release of OVA from poly(d,l-lactide-co-glycolide) (PLGA) nanoparticles [3]. Pegylated PLGA-based nanoparticles which were surface decorated with RGD molecules to target M cells (OVA association efficiency of 30-50\%) registered $5 \%$ release after $2 \mathrm{~h}$ of incubation in gastric medium $(\mathrm{HCl}$ $0.1 \mathrm{M}$ ) and $10-20 \%$ in intestinal medium. PLGA-lipid nanoparticles conjugated with ulex europaeus agglutinin-1 and containing the Toll-like receptor agonist monophosphoryl lipid A(95\% OVA association efficiency), released $17 \%$ of the protein after $2 \mathrm{~h}$ incubation in gastric acid medium $(0.1 \mathrm{M} \mathrm{HCl})$ and $3 \mathrm{~h}$ in intestinal medium (PBS pH 6.8) [64]. Comparatively, our work registered higher release of this protein after cumulative contact with the two media, but we expect that the remaining amount suffices for an adequate immunization effect.

While no studies report the release of OVA as model antigen from chitosan nanoparticles proposed for oral immunization, there are some works on chitosan-based microparticles. The results are however uneven, certainly as a result of different methods of preparation of microparticles and the use of diverse chitosan molecules. In fact, different works reported either immediate release of OVA in SGF [65], or only $50 \%$ in $2 \mathrm{~h}$ [66]. The latter behavior is similar to that registered in our work, where $45 \%$ released in $2 \mathrm{~h}$. The results regarding the release in SIF or PBS pH 7.3 were more coincident, where a maximum release of $10 \%-20 \%$ was determined after $1 \mathrm{~h}$ $[4,65,66]$. This is not far from the $3 \%$ registered in our work at the end of $4 \mathrm{~h}$.

\subsection{Safety evaluation of unloaded nanoparticles}

Caco- 2 cells were used to evaluate the safety profile of CS, LBGS and CS/LBGS nanoparticles by means of the metabolic assay MTT and the membrane integrity test based on LDH release. Samples were tested at $3 \mathrm{~h}$ and $24 \mathrm{~h}$, at concentrations between 0.1 and $1 \mathrm{mg} / \mathrm{mL}$. The overall observation of the results reveals a mild effect on cell viability from both the polymers and nanoparticles, considered to be devoid of biological relevance. At $3 \mathrm{~h}$ cell viability remained above $88 \%$ in all cases (data not shown). Prolonging the exposure until $24 \mathrm{~h}$ resulted in slight alterations (Fig. 5), although cell viability remained above $70 \%$ for all samples and concentrations. This value is that considered by ISO 10993-5 [67] as the level below which a toxic effect occurs. The only observation deserving a mention is that the exposure to the highest concentration tested $(1.0 \mathrm{mg} / \mathrm{mL})$ of CS decreased cell viability to around $70 \%(P<0.05)$, but nanoparticles shown $90 \%$ at the same concentration.

A control was used that consists in a mixture of DMEM and $\mathrm{H}_{2} \mathrm{O}$ in the same ratio used for the samples, taking into account that both the raw materials and the nanoparticles were solubilized/suspended in water and diluted with cell culture medium prior to incubation with the cells. This enables a real evaluation on the contribution of these materials on the final cell viability. As observed in Fig. 5, the cell viability induced by this control was $72 \%$ when tested at $24 \mathrm{~h}$, similar to that of $1.0 \mathrm{mg} / \mathrm{mL}$ of CS $(71 \%)$. Remarkably, focusing on the concentration of $1.0 \mathrm{mg} / \mathrm{mL}$, the cell viability obtained by exposure to the nanoparticles was higher than that registered for CS assessed individually and the control of DMEM $+\mathrm{H}_{2} \mathrm{O}(P<0.05)$.

LBG-based nanoparticles are now being reported for the first time. Therefore, the literature does not report any information regarding the cytotoxic effect of the particles, hindering the establishment of any comparison. In contrast, chitosan-based nanoparticles have been reported for a long time. As chitosan is one of the most used polymers in drug delivery, nanocarriers other than nanoparticles have been proposed, such as nanocapsules and nanoemulsions, for instance. This diversity affects viability results

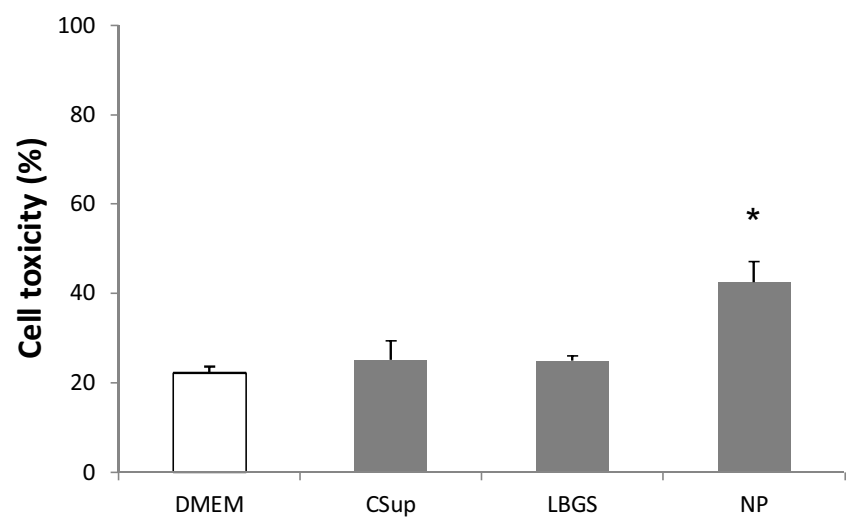

Fig. 6. Caco-2 cell viability measured by the LDH release assay after $24 \mathrm{~h}$ exposure to $1 \mathrm{mg} / \mathrm{mL}$ solutions of Chitosan (CS), sulfate Locust Bean Gum (LBGS) derivative and CS/LBGS nanoparticles (NP). Data represent mean $\pm S E M(n \geq 3$, three replicates per experiment). ${ }^{*} P<0.05$ compared with DMEM 
[30], but even when similar carriers are at play, it is frequently difficult to establish valid comparisons taking into account the large variety of chitosan molecules (chitosan base/salt(s), molecular weight, deacetylation degree, etc.) which is also known to have a role on cell viability [68]. Notwithstanding these limitations, the general outcome regarding chitosan-based nanoparticles is a very mild effect on Caco- 2 cell viability. Attempting to perform a direct and accurate comparison, other works assessing a nanoparticle concentration of $1.0 \mathrm{mg} / \mathrm{mL}$ reported similar results to those found in this work, both at $3 \mathrm{~h} \mathrm{[69]}$ and $24 \mathrm{~h} \mathrm{[70,71].}$

The assay of $\mathrm{LDH}$ release complements the information provided by the MTT. The loss of intracellular LDH and its release to the culture medium is an indicator of irreversible cell death due to cell membrane damage [72,73]. The amount of LDH released by Caco2 cells exposed to CS/LBGS nanoparticles was determined, using as control both the incubation with cell culture medium (negative control of cell death) and the exposure to a lysis buffer (positive control of cell death, assumed as 100\%). As observed in Fig. 6, the exposure to the raw materials CS and LBGS induced the release of an amount of $\mathrm{LDH}$ (around 25\%) that is comparable to that of the negative control, as no statistically significant differences were detected. On the contrary, the contact with CS/LBGS nanoparticles resulted in an unexpected increased level of LDH release (43\%; $P<0.05)$, which is indicative of cytotoxicity.

If a direct correlation between LDH release and cell death is assumed, the contact with the nanoparticles results in approximately $60 \%$ cell viability, comparing with the $80 \%$ elicited by DMEM. Surprisingly, this does not correspond with the observations resulting from the MTT assay, in which a cell viability of $90 \%$ was observed for this condition. One possible explanation is that these nanoparticles act as metabolic enhancers, thus although with a lower number of available cells (as indicated by the LDH assay), MTT conversion into formazan is accelerated, resulting in the overestimation of the cell viability. This difference in the results of the two assays reinforces the need to perform various and different tests to conclude on the safety profile of nanoparticles.

\subsection{In vivo evaluation of the immune response in $B A L B / c$ mice}

After verifying the ability of LBG derivatives to produce nanoparticles with capacity to associate antigens of interest and evaluating the cytotoxicity of the nanoparticles in an intestinal model, an in vivo assay was designed and setup to evaluate the adjuvant effect of the particles towards an immunization strategy.

A study was performed with HE-loaded CS/LBGS nanoparticles, but no differences were generally observed to a statistically significant level when comparing the results elicited by free HE and NP-HE (data not shown). HE antigens are possibly too robust and capable of inducing a strong immune response when administered in free form, thus not potentiating the observation of an adjuvant effect by the nanoparticles. This fact may be explained by the particulate nature of HE (liposome-like), and the high content of LPS in the HE extract. It is known that LPS is recognized by TLR-4 [74]. Nevertheless, long-term memory and challenge studies should be performed in order to verify whether NP-HE could improve the immunological response, in spite of a similar antibody-mediated response. After this approach with $\mathrm{HE}$, the need to test a second antigen was identified. The soluble antigen OVA was selected for this effect, being administered in free and encapsulated form (NPOVA), by oral and subcutaneous routes. Serum and feces from the mice were collected before the immunization and at weeks 1-6 post immunization. Unloaded nanoparticles were tested in the NPHE assay and, as expected, were shown to not induce any type of immune response (data not shown).

Figs. 7 and 8 show the serum titers of IgG1 and IgG2a (systemic response), and IgA (mucosal response) after an oral or subcu-
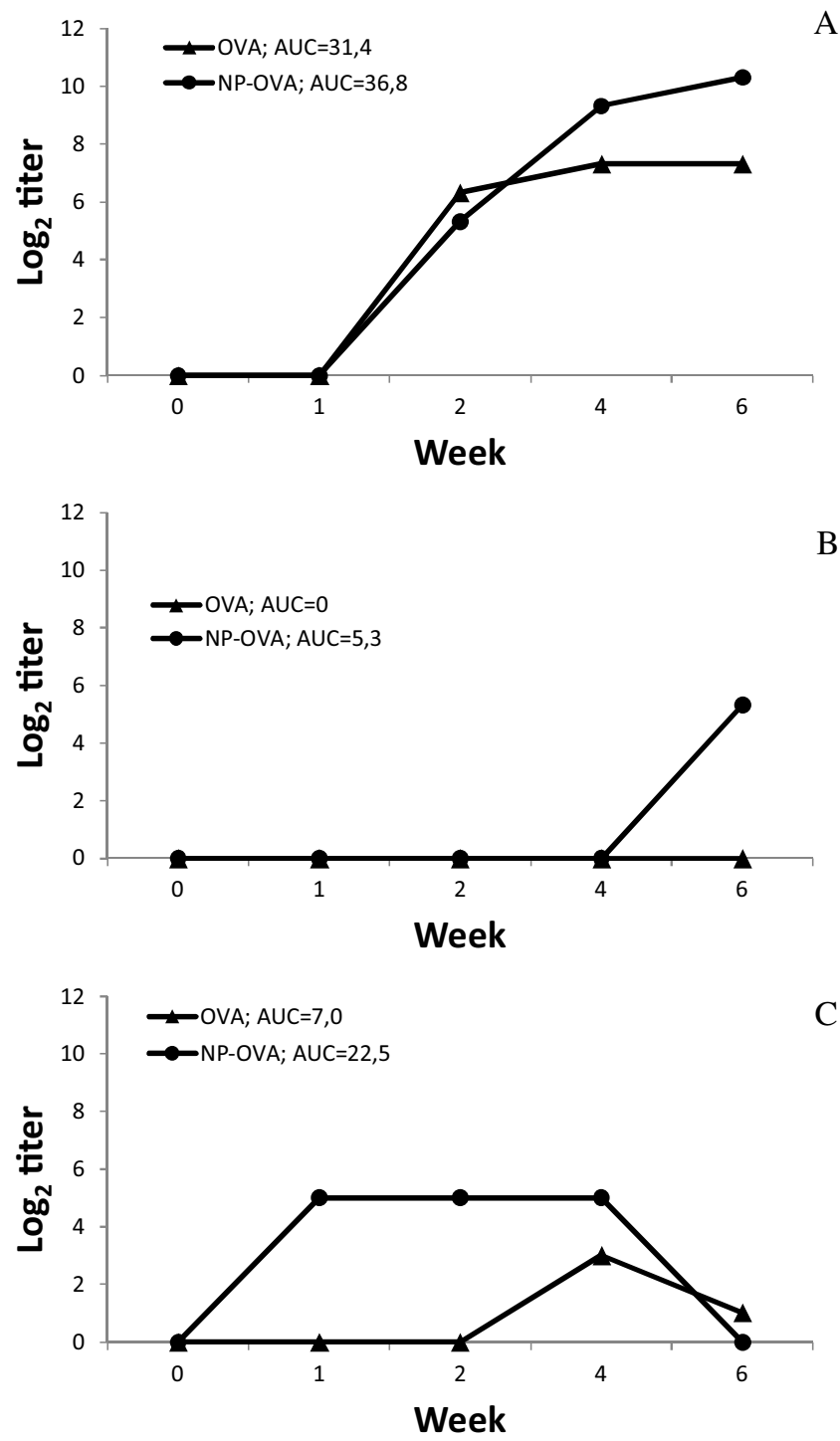

Fig. 7. Immunogenicity of OVA after oral administration in mice. Serum anti-OVA A) IgG1; B) IgG2a and C) faecal anti-OVA IgA response in BALB/c mice ( $n=6)$ after oral immunization with $100 \mu \mathrm{g}$ of OVA solution (OVA) or $100 \mu \mathrm{g}$ of encapsulated OVA (NP-OVA). Antibody titers were determined in pooled serum samples at days $0,7,14,21$ and 28 post-administration.

taneous immunization of mice by a single dose of OVA-loaded nanoparticles or free OVA. Overall, it is observed the adjuvant effect of nanoparticle formulations compared to free OVA in both routes. Focusing on the oral immunization (Fig. 7), the elicited IgG1 specific response (Th2) was significant after OVA immunization, either free or encapsulated. Nanoparticles did not improve the effect of OVA regarding Th2 activation (IgG1, Fig. 7A), but, however, a strong improvement was observed regarding Th1 activation (IgG2a, Fig. 7B), as the respective area under the curve (AUC) was five times higher than that determined for free OVA. Intestinal immune system has a predisposition towards Th2 cell responses since antigen presentation by DCs from PPs are characterized by the production of IL-4, IL-6, and IL-10, which inhibit a Th1 response [75]. It is also important to refer that soluble antigens usually elicit high levels of IgG1 antibodies (Th2 response), but very low levels of IgG2a (Th1 response) [76]. Therefore, under these circumstances, the role of the adjuvant becomes critical in order to achieve a more balanced Th1/Th2 response. A similar positive effect of association was 

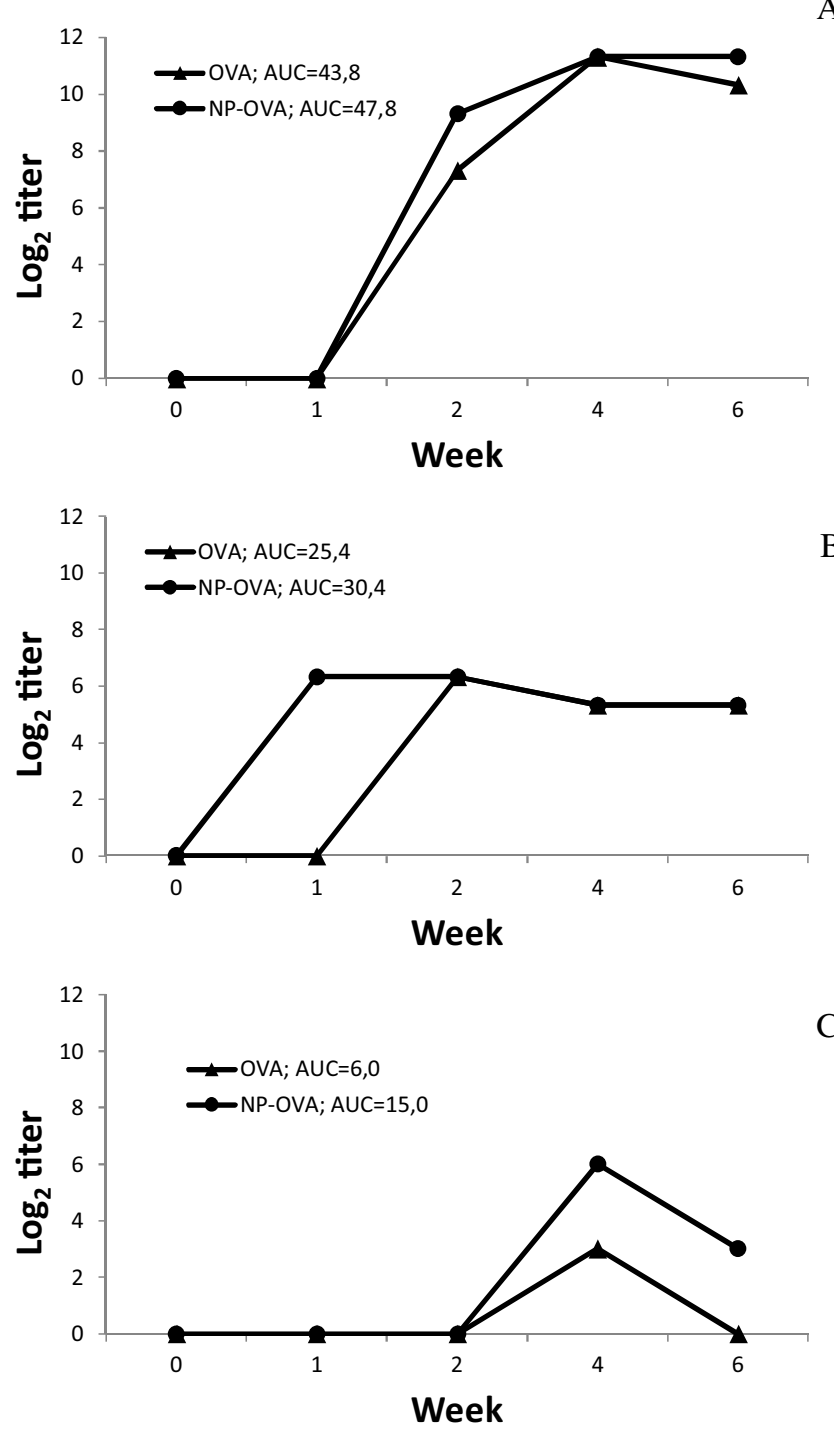

Fig. 8. Immunogenicity of OVA after S.C. administration in mice. Serum anti-OVA A) IgG1; B) IgG2a and C) faecal anti-OVA IgA response in BALB/c mice $(n=6)$ after S.C. immunization with $20 \mu \mathrm{g}$ of OVA solution (OVA) or $20 \mu \mathrm{g}$ of encapsulated OVA (NP-OVA). Antibody titers were determined in pooled faecal samples at days 0,7 , 14,21 and 28 post-administration.

apparent regarding the mucosal response (IgA), as shown in Fig. 7C. In this respect, OVA-loaded nanoparticles registered a 3-fold higher AUC when comparing with free OVA.

When considering the determined AUC, a comparable mucosal response was elicited when the formulation was administered by subcutaneous route (Fig. 8C). However, the pattern registered for the responses was markedly different. In fact, while the oral immunization induced a response that was detected from week one, the response obtained upon subcutaneous immunization was only observed starting from week two to four. In turn, the subcutaneous administration of nanoencapsulated OVA showed a similar activation of Th2 (IgG1, Fig. 8A) and Th1 (IgG2a, Fig. 8B) to that described for free OVA.

After oral immunization, OVA-loaded nanoparticles showed a predominant Th2 response compared to the slightly elicited Th1 one $\left(\mathrm{AUC}_{\mathrm{Th} 2}\right.$ was seven times higher than $\mathrm{AUC}_{\mathrm{Th} 1}$ ), consistent with results obtained by others [53,77-80]. On the contrary, subcutaneous immunization with OVA-loaded nanoparticles elicited a more balanced Th1 and Th2 response. In order to perform a semiquantitative comparison between the nanoparticle formulation and the free OVA, the AUC of the titers representing the systemic immune response $\left(\mathrm{AUC}_{\mathrm{Th} 1}, \mathrm{AUC}_{\mathrm{Th} 2}\right.$ ) were measured and summed. Regarding the oral immunization, an increase of $34 \%$ in the total AUC was accounted for the encapsulated OVA. In turn, this association only induced a $13 \%$ increase in the response after subcutaneous immunization. This is possibly justified by the presence of mannose units in LBG nanoparticles, potentiating a stronger interaction with $\mathrm{M}$ cells and the mediation of a stronger immune response when the nanoparticles are administered by the oral route, as was suggested in a study performed with mannosylated nanoparticles $[80,81]$. In that work it was verified that mannosylated nanoparticles induced a more balanced Th1/Th2 response comparing with non-mannosylated nanoparticles, an effect attributed to the high tropism of mannosylated particles for uptake by PPs rich in APCs [63].

The mucosal IgA antibodies obtained after oral immunization with OVA-loaded nanoparticles were higher than after subcutaneous administration. This phenomenon may be related to the effective uptake of nanoparticles by gut Peyer's patches, obviously only possible after oral delivery, and the passage of the particulate system to lymphocytes causing an effective generation of mucosal IgA. However, it should be taken into account that a 5-fold higher dose of antigen was administered by oral route.

The described results indicate a significant adjuvant effect of CS/LBGS nanoparticles. Both the presence of mannose units in LBG, which target M cells and DC's, and the mucoadhesive characteristics of CS, are thought to have played a role in the improved response mediated by nanoparticles. Additionally, results obtained by others suggest that targeting to M cells and/or mucosal DCs should be the way to achieve a better and/or more balanced immune response $[53,77-80]$. It is worth to mention that the oral immunization performed in these in vivo assays, truly evaluates the system capability, since it was made by oral gavage (not intraduodenally) and no prior administration of sodium bicarbonate solution, in order to neutralize the acid environment of the stomach, was made. The role of $\mathrm{M}$ cells and mucosal DCs to achieve the adjuvant properties suggested herein should be demonstrated.

\section{Conclusions}

This work reports for the first time the production of nanoparticles based on locust bean gum, demonstrating the ability of the produced sulfate derivative of locust bean gum to form nanoparticulate complexes with chitosan by means of an electrostatic interaction. The produced nanoparticles were proposed as adjuvants in oral immunization, efficiently associating two model antigens (HE antigenic complex and OVA) without compromising their structural integrity. Displaying a size around $180-200 \mathrm{~nm}$ and a positive zeta potential, the antigen-loaded nanoparticles were deemed adequate for the proposed application in oral immunization. The cytotoxic assessment performed in Caco-2 cells revealed no alterations at the level of cell metabolic activity upon exposure to the nanoparticles, but a mild negative effect on cell membrane integrity was observed. The in vivo proof of concept demonstrated the adjuvant effect of the proposed system when the soluble antigen OVA was used as model. Additionally and as expected, nanoparticles were capable of inducing not only a systemic response, but also a response at the mucosal level.

Summing up, CS/LBGS nanoparticles are promising as an antigen delivery strategy. However, further experiments are required in order to define its application on immune modulation. 


\section{Acknowledgements}

This work was supported by national Portuguese funding through FCT - Fundação para a Ciência e a Tecnologia, project ref. PTDC/SAU-FCF/100291/2008 and PEst-OE/EQB/LA0023/2011.

This work was also financed by FEDER - Fundo Europeu de Desenvolvimento Regional funds through the COMPETE 2020 Operacional Programme for Competitiveness and Internationalisation (POCI), Portugal 2020, and by Portuguese funds through FCT - Fundação para a Ciência e a Tecnologia/Ministério da Ciência, Tecnologia e Inovação in the framework of the project "Institute for Research and Innovation in Health Sciences" (POCI-01-0145FEDER-007274).

\section{References}

[1] L.A. Caetano, A.J. Almeida, L.M.D. Gonçalves, J. Biomed. Nanotechnol. 10 (2014) 2295-2316.

[2] C.J.H. Davitt, E.C. Lavelle, Adv. Drug Deliv. Rev. 91 (2015) 52-69.

[3] M. Garinot, V. Fievez, V. Pourcelle, F. Stoffelbach, A. des Rieux, L. Plapied, I. Theate, H. Freichels, C. Jerôme, J. Marchand-Brynaert, Y.J. Schneider, V. Preat, J. Control. Release 120 (2007) 195-204.

[4] I.M. van der Lubben, J.C. Verhoef, G. Borchard, H.E. Junginger, Adv. Drug Deliv Rev. 52 (2001) 139-144.

[5] E.C. Lavelle, D.T. O'Hagan, Expert Opin. Drug Deliv. 3 (2006) 747-762.

[6] M. Yamamoto, D.W. Pascual, H. Kiyono, M cell-targeted mucosal vaccine strategies, in: A.P. Kozlowski (Ed.), Mucosal Vaccines: Modern Concepts, Strategies, and Challenges, Springer Berlin Heidelberg, Berlin, Heidelberg, 2012, pp. 39-52.

[7] J.F. Mann, E. Shakir, K.C. Carter, A.B. Mullen, J. Alexander, V.A. Ferro, Vaccine 27 (2009) 3643-3649.

[8] P.L. Ogra, H. Faden, R.C. Welliver, Clin. Microbiol. Rev. 14 (2001) 430-445.

[9] M.E. Baca-Estrada, M. Foldvari, S.L. Babiuk, L.A. Babiuk, J. Biotechnol. 83 (2000) 91-104.

[10] M. Kristensen, H.M. Nielsen, Basic Clin. Pharmacol. Toxicol. 118 (2016) 99-106.

[11] V. Truong-Le, P.M. Lovalenti, A.M. Abdul-Fattah, Adv. Drug Deliv. Rev. 93 (2015) 95-108

[12] R. Sharma, U. Agrawal, N. Mody, S.P. Vyas, Biotechnol. Adv. 33 (2015) 64-79.

[13] C. Gamazo, N. Martín-Arbella, A. Brotons, A.I. Camacho, J.M. Irache, Eur J. Pharm. Biopharm. 96 (2015) 454-463.

[14] M. Alonso-Sande, D. Teijeiro-Osorio, C. Remuñán-López, M.J. Alonso, Eur. J. Pharm. Biopharm. 72 (2009) 453-462.

[15] H. Tomizawa, Y. Aramaki, Y. Fujii, T. Hara, N. Suzuki, K. Yachi, H. Kikuchi, S. Tsuchiya, Pharm. Res. 10 (1993) 549-552.

[16] M. Dionísio, A. Grenha, J. Pharm. Bioallied Sci. 4 (2012) 75-85.

[17] E. Weir, K. Hatch, CMAJ: Can. Med. Assoc. J. 171 (2004), 1050-1050.

[18] F. Sarti, G. Perera, F. Hintzen, K. Kotti, V. Karageorgiou, O. Kammona, C. Kiparissides, A. Bernkop-Schnurch, Biomaterials 32 (2011) 4052-4057.

[19] S. Chadwick, C. Kriegel, M. Amiji, Adv. Drug Deliv. Rev. 62 (2010) 394-407.

[20] J. Ochoa-Repáraz, B. Sesma, M. Álvarez, M.J. Renedo, J.M. Irache, C. Gamazo, Vet. Res. 35 (2004) 291-298.

[21] J. Ochoa, J.M. Irache, I. Tamayo, A. Walz, V.G. DelVecchio, C. Gamazo, Vaccine 25 (2007) 4410-4419.

[22] Y. Kawamura, in, Joint FAO/WHO Expert Committee on Food Additives (2008)

[23] N. Bouzouita, A. Khaldi, S. Zgoulli, L. Chebil, R. Chekki, M.M. Chaabouni, P. Thonart, Food Chem. 101 (2007) 1508-1515

[24] F. Wang, Y.J. Wang, Z. Sun, J. Food Sci. 67 (2002) 2609-2614.

[25] H. Yuan, W. Zhang, X. Li, X. Lu, N. Li, X. Gao, J. Song, Carbohydr. Res. 340 (2005) 685-692.

[26] USP, The United States Pharmacopeia XXXII/National Formulary XXVII, The United States Pharmacopeial Convention, Rockville (2009).

[27] J. Carmichael, W.G. DeGraff, A.F. Gazdar, J.D. Minna, J.B. Mitchell, Cancer Res. 47 (1987) 936-942.

[28] L. Braydich-Stolle, S. Hussain, J.J. Schlager, M.C. Hofmann, Toxicol. Sci. 88 (2005) 412-419.

[29] A. Grenha, J. Drug Target. 20 (2012) 291-300.

[30] C. Prego, D. Torres, M.J. Alonso, Expert Opin. Drug Deliv. 2 (2005) 843-854.

[31] E. Fernandez-Megia, R. Novoa-Carballal, E. Quiñoá, R. Riguera, Carbohydr. Polym. 61 (2005) 155-161.

[32] S. Rodrigues, A.M. da Costa, A. Grenha, Carbohydr. Polym. 89 (2012) 282-289.

[33] S. Rodrigues, L. Cardoso, A. da Costa, A. Grenha, Materials 8 (2015) 5647-5670

[34] Z. Li, L. Gu, J. Agric. Food Chem. 59 (2011) 4225-4231.

[35] M. Dionísio, L. Braz, M. Corvo, J.P. Lourenço, A. Grenha, A.M.R. da Costa, Int. J. Biol. Macromol. 86 (2016) 129-138

[36] M. Dionísio, C. Cordeiro, C. Remuñán-López, B. Seijo, A.M. Rosa da Costa, A. Grenha, Eur. J. Pharm. Sci. 50 (2013) 102-113.
[37] M. Müller, B. Keßler, J. Fröhlich, S. Poeschla, B. Torger, Polymers 3 (2011) $762-778$

[38] V. Starchenko, M. Müller, N. Lebovka, J. Phys. Chem. C 112 (2008) 8863-8869. [39] M. de la Fuente, B. Seijo, M.J. Alonso, Macromol. Biosci. 8 (2008) 441-450.

[40] D. Teijeiro-Osorio, C. Remunan-Lopez, M.J. Alonso, Biomacromolecules 10 (2009) 243-249.

[41] A. Grenha, M.E. Gomes, M. Rodrigues, V.E. Santo, J.F. Mano, N.M. Neves, R.L. Reis, J. Biomed. Mater. Res. A 92 A (2010) 1265-1272.

[42] R. Fernández-Urrusuno, D. Romani, P. Calvo, J. Vila-Jato, M. Alonso, STP Pharma Sci. 9 (1999) 429-436.

[43] M.A. Bonafonte, C. Solano, B. Sesma, M. Alvarez, L. Montuenga, D. García-Ros, C. Gamazo, FEMS Microbiol. Lett. 191 (2000) 31-36.

[44] D. Werling, T.W. Jungi, Vet. Immunol. Immunopathol. 91 (2003) 1-12.

[45] P. Calvo, C. Remunan-Lopez, J.L. Vila-Jato, M.J. Alonso, Pharm. Res. 14 (1997) 1431-1436.

[46] B. Sarmento, A. Ribeiro, F. Veiga, D. Ferreira, Colloids Surf. B: Biointerfaces 53 (2006) 193-202.

[47] A. Grenha, B. Seijo, C. Remuñán-López, Eur. J. Pharm. Sci. 25 (2005) 427-437.

[48] M.P. Desai, V. Labhasetwar, G.L. Amidon, R.J. Levy, Pharm. Res. 13 (1996) 1838-1845.

[49] P. Jani, G.W. Halbert, J. Langridge, A.T. Florence, J. Pharm. Pharmacol. 42 (1990) 821-826.

[50] C.-M. Lehr, J.A. Bouwstra, E.H. Schacht, H.E. Junginger, Int. J. Pharm. 78 (1992) 43-48.

[51] H. Le Buanec, C. Vetu, A. Lachgar, M.A. Benoit, J. Gillard, S. Paturance, J. Aucouturier, V. Gane, D. Zagury, B. Bizzini, Biomed. Pharmacother. 55 (2001) $316-320$.

[52] O. Borges, J. Tavares, A. de Sousa, G. Borchard, H.E. Junginger, A Cordeiro-da-Silva, Eur. J. Pharm. Sci. 32 (2007) 278-290.

[53] B. Slütter, L. Plapied, V. Fievez, M.A. Sande, A.d. Rieux, Y.-J. Schneider, E.V. Riet, W. Jiskoot, V. Préat, J. Control. Release 138 (2009) 113-121.

[54] A. Farhadian, N.M. Dounighi, M. Avadi, Hum. Vaccine Immunother. 11 (2015) 2811-2818.

[55] M. Abkar, A.S. Lotfi, J. Amani, K. Eskandari, M.F. Ramandi, J. Salimian, G.N. Brujeni, S. Alamian, M. Kamali, H. Koushki, Vet. Res. Commun. 39 (2015) 217-228

[56] B. Malik, A.K. Goyal, T.S. Markandeywar, G. Rath, F. Zakir, S.P. Vyas, J. Drug Target. 20 (2012) 76-84.

[57] N. Mishra, K. Khatri, M. Gupta, S.P. Vyas, Artif. Cells Nanomed. Biotechnol, 42 (2014) 245-255

[58] H. Harde, A.K. Agrawal, S. Jain, Pharm. Res. 32 (2015) 122-134.

[59] S. Dehghan, M.T. Kheiri, M. Tabatabaiean, S. Darzi, M. Tafaghodi, Arch. Pharm. Res. 36 (2013) 981-992.

[60] X.Y. Li, X. Li, X.Y. Kong, S. Shi, G. Guo, J. Zhang, F. Luo, X. Zhao, Y.Q. Wei, Z.Y. Qian, L. Yang, J. Nanosci. Nanotechnol. 10 (2010) 4850-4858.

[61] B. Sayın, S. Somavarapu, X.W. Li, M. Thanou, D. Sesardic, H.O. Alpar, S. Senel, Int. J. Pharm. 363 (2008) 139-148.

[62] F. Sarei, N.M. Dounighi, H. Zolfagharian, P. Khaki, S.M. Bidhendi, Indian J. Pharm. Sci. 75 (2013) 442-449.

[63] H.H. Salman, C. Gamazo, M.A. Campanero, J.M. Irache, J. Control. Release 106 (2005) 1-13.

[64] T. Ma, L. Wang, T. Yang, G. Ma, S. Wang, Int. J. Pharm. 473 (2014) 296-303.

[65] M. Hori, H. Onishi, Y. Machida, Int. J. Pharm. 297 (2005) 223-234.

[66] K. Maculotti, E.M. Tira, M. Sonaggere, P. Perugini, B. Conti, T. Modena, F, Pavanetto, J. Microencapsul. 26 (2009) 535-543.

[67] ISO, in, International Organization for Standardization (2009).

[68] C. Prego, D. Torres, M.J. Alonso, J. Nanosci, Nanotechnology 6 (2006) 1-8.

[69] L.C. da Silva, T. Garcia, M. Mori, G. Sandri, M.C. Bonferoni, P.V. Finotelli, L.P. Cinelli, C. Caramella, L.M. Cabral, Int. J. Nanomed. 7 (2012) 2975-2986.

[70] J.W. Loh, M. Saunders, L.-Y. Lim, Toxicol. Appl. Pharmacol. 262 (2012) 273-282.

[71] N.M. Zaki, M.M. Hafez, AAPS Pharm. Sci. Technol. 13 (2012) 411-421.

[72] G. Fotakis, J.A. Timbrell, Toxicol. Lett. 160 (2006) 171-177.

[73] A.J. Racher, D. Looby, J.B. Griffiths, Cytotechnology 3 (1990) 301-307.

[74] M. Gi, W. Im, S. Hong, Sensors 9 (2009) 6730-6751.

[75] R.L. Jump, A.D. Levine, J. Immunol. 168 (2002) 6113-6119.

[76] I. Gutierro, R.M. Hernández, M. Igartua, A.R. Gascón, J.L. Pedraz, Vaccine 21 (2002) 67-77.

[77] V. Fievez, L. Plapied, A.d. Rieux, V. Pourcelle, H. Freichels, V. Wascotte, M.-L. Vanderhaeghen, C. Jerôme, A. Vanderplasschen, J. Marchand-Brynaert, Y.-J. Schneider, V. Préat, Eur. J. Pharm. Biopharm. 73 (2009) 16-24.

[78] H.H. Salman, C. Gamazo, M. Agueros, J.M. Irache, Vaccine 25 (2007) 8123-8132.

[79] H.H. Salman, C. Gamazo, P.C. de Smidt, G. Russell-Jones, J.M. Irache, Pharm. Res. 25 (2008) 2859-2868.

[80] H.H. Salman, J.M. Irache, C. Gamazo, Vaccine 27 (2009) 4784-4790.

[81] J.M. Irache, H.H. Salman, S. Gomez, S. Espuelas, C. Gamazo, Front. Biosci. (Schol. Ed.) 2 (2010) 876-890. 